antibodies (AECA). Results of the mentioned tests were statistically evaluated.

Results

- No association has been found between presence of neurological symptoms and disease activity in patients with systemic lupus erythematosus

- Neurological symptoms were found significantly more frequently in subjects with high titer of $\operatorname{IgG}$ anticardiolipin antibodies and in patients with secondary antiphospholipid syndrome.

- There was a tendency for neurological symptoms to occur in patients with vascular complications.

Conclusion

\section{FRI0131 INCIDENCE OF RENAL FLARES AND THEIR PROGNOSTIC SIGNIFICANCE IN DIFFUSE PROLIFERATIVE GLOMERULONEPHRITIS}

${ }^{1} \mathrm{M}$ Mosca, ${ }^{1} \mathrm{~W}$ Bencivelli, ${ }^{1} \mathrm{R}$ Neri, ${ }^{2} \mathrm{~A}$ Pasquariello, ${ }^{2} \mathrm{R}$ Puccini, ${ }^{2} \mathrm{~V}$ Batini, ${ }^{1} \mathrm{~S}$ Bombardieri. ${ }^{1}$ Rheumatology Unit; ${ }^{2}$ Nephrology, University of Pisa, Pisa, Italy

10.1136/annrheumdis-2001.166

Background Diffuse Proliferative Glomerulonephritis (DPGN) is considered one of the most important factors influencing the long term prognosis of SLE patients. Renal flares are considered important risk factors influencing the outcome of renal involvement.

Objectives The aim of this study was to evaluate the occurrence of renal flares and their prognostic significance for the renal outcome in a population of SLE patients with DPGN.

Methods The clinical, laboratory and histological data of 91 SLE patients ( 8 males and 83 females) followed at the Rheumatology Unit of the University of Pisa, were retrospectively evaluated.

Results Fourty-nine patients (54\%) presented a renal flare a mean of 42 months after the renal biopsy. Twenty-eight flares $(57 \%)$ were proteinuric, twenty-one $(43 \%)$ were nephritic flares. A young age at the time of the renal biopsy and treatment with steroids alone, were significantly correlated with the occurrence of renal flares, furthermore a high Activity Index (AI >9) and the presence of karyorrhexis on renal histology were correlated with the occurrence of nephritic flares. A poor renal outcome occurred in $27 \%$ of the patients; serum creatinine levels at the time of renal biopsy, the presence of karyorrhexis and the composite Chronicity Index (CI) as well as all of its component on renal histology, the number of renal flares (both proteinuric and nephritic), the occurrence of nephritic flares and early proteinuric flares (i.e. in the first 17 months after the renal biopsy), were correlated with a poor renal outcome.

Conclusion In conclusion, the analysis of our patients has showed that nephritic flares and early proteinuric flares are important risk factors for the occurrence of a poor renal outcome. Since young patients with an elevated AI and karyorrhexis are at increased risk for renal flares, they should be candidated for a more aggressive and prolonged treatment.

\section{REFERENCES}

1 Moroni G, et al. Kidney Int. 1996;50:2047-53

2 Ponticelli C, et al. Lupus 1998;7:635-8

3 Ciruelo, et al. Arthritis Rheum. 1996;39:2028-34
FRI0132 DECREASED SERUM DNASE 1 ACTIVITY LEVELS IN SLE PATIENTS WITH ACTIVE DISEASE

${ }^{1}$ I Gunnarsson, ${ }^{1} \mathrm{~F}$ Guo-Zhong, ${ }^{2} \mathrm{HG}$ Mannherz, ${ }^{2} \mathrm{M}$ Napirei, ${ }^{2} \mathrm{~A}$ Gültekin, ${ }^{1} \mathrm{~J}$ Frostegård. ${ }^{1}$ Department of Rheumatology, Karolinska Hospital, Stockholm, Sweden; ${ }^{2}$ Abteilung Für Anatomie and Embryologie, Medizinishe Fakultät, Ruhr-Universität Bochum, Bochum, Germany

10.1136/annrheumdis-2001.167

Background DNASE 1 (deoxyribonuclease 1; EC 3.1.21.1) deficient mice have been shown to develop classical symptoms of Systemic Lupus Erythematosus (SLE) with anti-nuclear antibodies in the circulation. Low serum DNASE 1 activity has been reported in patients with SLE.

Objectives The aim of this study was to investigate serum DNASE 1 activity in SLE patients in relation to disease activity. In addition, the relationship between the DNASE 1 activity and renal involvement was analysed.

Methods Serum samples from fifty patients with SLE (ACR criteria), 39 females and 11 males, mean age 44, were analysed for DNASE 1 activity by the single radial enzyme-diffusion method (SRED) (Nadano et al., 1993). All sera were analysed on three separate occasions and the mean value $(\mathrm{pg} / \mathrm{ml})$ from the three experiments was used. Anti-dsDNA antibodies were detected by indirect IFL. SLE disease activity was assessed according to the Systemic Lupus Activity Measure (SLAM). A SLAM score of 7 or above was considered as high. Thirty $(60 \%)$ had high disease activity (SLE/active), the other 20 patients (SLAM <7) were classified as SLE/inactive. Twenty-six $(52 \%)$ of the 50 SLE patients had glomerulonephritis, in all cases confirmed by renal biopsy; the other 24 patients (48\%) had no clinical signs of renal involvement. The renal biopsies were classified according to the WHO classification. Six patients had WHO class II, 12 WHO class III/IV and 8 WHO class V.

Results Serum DNASE 1 levels were significantly lower in the SLE/active patient group as compared to the SLE/inactive patients $(12.4 \pm 3.8$ vs. $16.1 \pm 3.7 \mathrm{pg} / \mathrm{ml}$, mean $\pm S D, p<$ 0.005). Among the $20 \mathrm{SLE} /$ inactive patients, a trend towards lower serum DNASE 1 levels was noted in patients with renal involvement $(\mathrm{n}=9)(14.8 \pm 3.6 \mathrm{pg} / \mathrm{ml})$ as compared to patients without renal involvement $(\mathrm{n}=11)(17.1 \pm 3.5 \mathrm{pg} / \mathrm{ml}, \mathrm{p}=$ 0.06). However, in SLE/active patients, there was no significant difference in DNASE 1 levels between patients with and without renal involvement. There was no association between the levels of DNASE 1 activity and anti-dsDNA antibody titres. Twentyfive of the patients received prednisolone treatment. Among these patients, low levels of serum DNASE 1 activity were found to be associated with high dose prednisolone therapy $(\mathrm{r}=$ $0.552, \mathrm{p}<0.005)$.

Conclusion This study demonstrated low DNASE 1 levels to be associated with high disease activity in SLE patients and may represent a novel marker of disease activity independently of anti-dsDNA antibodies. Further studies are needed to clarify the role of DNASE 1 in SLE manifestations and disease development. 